\title{
ORGANIZACION ESPACIAL DE SEDIMENTOS EN LAS BARRAS DEL CAUCE TRENZADO DEL RIO OJA
}

\author{
Amelia Gómez Villar* \\ Ricardo Martínez Castroviejo**
}

\section{RESUMEN}

\begin{abstract}
En este artículo se definen diferentes ambientes deposicionales atendiendo al eje mayor de los sedimentos en tres puntos del cauce trenzado del río Oja: dos en el sector de la Sierra de la Demanda y un tercero en el tramo de los conglomerados de borde de cuenca, cerca de Santurde. Los resultados muestran que el tamaño medio de los sedimentos sobre las barras y canales decrecen aguas abajo y las irregularidades están definidas por los procesos locales de transporte de sedimentos.
\end{abstract}

\section{SUMMARY}

In this paper we have defined different depositional environments atending to the longest axis of sediments in three points of river Oja braided channel: two in the Sierra de la Demanda and one third in the basin border conglomerates section, near Santurde. The results show that the average size of sediments on the bars and channels decreases downstream and the irregularities are defined for the local sediment transport processes.

PALABRAS CLAVES: río Oja, cauce trenzado, barras de sedimentos.

KEY WORDS: river Oja, braided channel, sediment bars.

* Departamento de Geografía y Ordenación del Territorio. Colegio Universitario de La Rioja.

** Instituto Pirenaico de Ecología (C.S.I.C.). Jaca. Huesca. 


\section{GOMEZ Y MARTINEZ}

Los canales aluviales constituyen elementos muy importantes en la interpretación del paisaje y han sido ampliamente trabajados en áreas de latitudes medias (LEOPOLD et al, 1964; GREGORY y WALLING, 1976; DUNNE y LEOPOLD, 1978). De ellos, los cauces trenzados (braided stream) son los menos intensamente tratados (GREGORY y WALLING, 1976) -aunque el mecanismo de trenzamiento (ALLEN, 1965) ha sido estudiado en el campo por STRAHLER (1951), KRIGSTROM (1962) y FAHNESTOCK (1963) y en laboratorio por LEOPOLD y WOLMAN (1957) - y los más complejos de definir; MIALL (1977) considera que los sistemas trenzados son cauces con múltiples canales, mientras que para LEOPOLD y WOLMAN (1957) son ríos que fluyen en dos, tres o más canales anastomosados y SCHUMM (1968) afirma que son ríos con carga de fondo con un canal único, los cuales en aguas bajas tienen islas de sedimentos e islas con vegetación relativamente permanente.

Esas acumulaciones sedimentarias en forma de islas y barras son el mejor rasgo distintivo de la morfología de ríos trenzados y sirven como disipadores de energía que permiten la configuración de canales relativamente estables para transportar sedimentos (CHURCH y JONES, 1982). Presentan una morfología en superficie muy variada -barras longitudinales, puntuales, transversales, etc.- y una dinámica diferente. Han sido ampliamente estudiadas, entre otros, por DOEGLAS (1962), KRIGSTROM (1962), RUST (1972), MIALL (1977), BLUCK (1974, 1982), CHURCH y JONES (1982), DAWSON (1987), GUPTA y DUTT (1989).

El cauce del río Oja, a pocos kilómetros de su nacimiento, inicia un modelo trenzado con canales de diversa anchura y profundidad y una gran densidad de barras e islas de sedimentos. El reconocimiento y estudio de estas estructuras permite la reconstrucción cualitativa de las condiciones hidráulicas e hidrodinámicas de un río trenzado. El objetivo que se persigue en este trabajo es analizar la organización espacial superficial de los sedimentos en los canales y barras del río Oja teniendo en cuenta su morfología y evolución.

\section{METODOS}

Para cumplir este objetivo hemos seleccionado tres puntos en el cauce del río en los Km. 12 (Zaldierna), 15 (Ezcaray) y 25 (Santurde) respectivamente. La elección de los mismos ha estado determinada por dos razones:

- Son tres sectores escasamente alterados por el hombre

- Aguas arriba del Km. 12 el trenzamiento es muy incipiente y aguas abajo del Km. 25 la continua extracción de áridos, construcción de diques en las márgenes, etc., supone una destrucción sistemática del cauce.

Con objeto de analizar la morfología del cauce en esos puntos se ha realizado una cartografía detallada de los canales y principales figuras deposicionales que aparecen, destacando especialmente la distribucción superficial de los sedimentos y direcciones de flujo en los canales y en las barras de sedimentos, a la vez que se ha medido la longitud de cada barra y se ha tomado la pendiente media en los tres puntos. Para observar la evolución del cauce se ha calculado la anchura del mismo y se han realizado tres transeptos perpendiculares al flujo de la corriente con pantómetro de Pitty de $25 \mathrm{~cm}$., uno para cada uno de los tres puntos de estudio en su sector central. Con la información recogida se han trazado tres perfiles transversales en donde quedan reflejadas las variaciones en la profundidad y anchura de los canales principales y secundarios y desarrollo vertical de las barras de sedimentos. 


\section{ORGANIZACION DE SEDIMENTOS EN BARRAS}

De los tres métodos de muestreo de sedimentos fluviales -con cuadrícula, al azar y a peso- (SEGURA, 1987), se ha usado el muestreo al azar de WOLMAN, (1954). Cada $20 \mathrm{~cm}$. de distancia se ha medido en milímetros el eje mayor de 100 cantos en los canales y en la punta, cola, centro y márgenes laterales de las barras de sedimentos, siendo en total 26 los sectores muestreados. Mediante un tratamiento estadístico con ordenador se han elaborado histogramas de frecuencias y se ha calculado el tamaño medio de los sedimentos y la desviación estándar para cada sector con el fin de analizar la evolución del tamaño de cantos en distintos ambientes dentro del cauce.

Finalmente, con ayuda de la fotografía aérea de 1978 -escala 1:18.000 - se ha calculado un índice de trenzamiento estimando la longitud de todos los canales o segmentos del cauce trenzado por cada uno de los tres kilómetros -en línea recta- seleccionados; en realidad se trata de un indice de sinuosidad.

\section{AREA DE ESTUDIO}

La cuenca del río Oja (Fig. 1) ocupa una extensión de $380 \mathrm{Km}^{2}$ y se localiza en el sector más noroccidental del Sistema Ibérico. Su cabecera y curso alto se inscriben en el macizo paleozoico de la Sierra de la Demanda, compuesto por una litología a base de cuarcitas, pizarras y esquistos; el curso medio y bajo circula entre los materiales terciarios de la Depresión del Ebro, atravesando primero los conglomerados de borde de cuenca de facies Santurdejo (RIBA, 1955) y areniscas y arcillas hacia la desembocadura. Entre ambos sectores cruza una banda de materiales mesozoicos compuesta por calizas, areniscas, arcillas y conglomerados de Triásico y Jurásico.

El cauce del río Oja, que tiene una longitud de $48,55 \mathrm{Km}$. presenta un cambio progresivo desde la cabecera hacia su salida a la Depresión. Poco después de su nacimiento circula entre laderas muy empinadas que muestran una gran estabilidad y posee un curso de meandros encajados -el control estructural domina el trazado del cauce- con fuerte pendiente $(25,8 \%)$ que permite la evacuación de sedimentos y la incisión en el propio lecho a la vez que dificulta el ensanchamiento del cauce. Existe un equilibrio entre la capacidad de transporte y volumen/tamaño de sedimentos aportados desde las laderas (GARCIA-RUIZ et al, 1987).

Pocos kilómetros después presenta un trazado rectilíneo en un corto tramo y a partir del Km. 11 sufre un cambio espectacular. El canal único inicial se divide en varios brazos entrecruzados, inestables, dejando entre si obstáculos y barras de sedimentos. El fenómeno, incipiente al principio se acentúa aguas abajo. Los barrancos que afluyen al río Oja, con fuertes pendientes, aportan al cauce principal una gran cantidad de materiales procedentes de unas laderas con una dinámica muy activa -desprendimientos y cabeceras activas de barrancos-; este hecho coincide con un descenso importante en la pendiente longitudinal del río (inferior al $4 \%$ ), lo que supone una pérdida de velocidad del agua, y un aumento en la anchura del cauce. Se produce un desequilibrio importante entre la enorme cantidad de sedimentos localizados en el cauce y la competencia del flujo para evacuarlos. Este desequilibrio se compensa con un cambio en las condiciones hidrodinámicas del río que son las que explican la aparición de ese modelo trenzado, muy bien desarrollado en todo el sector de la Depresión del Ebro.

El modelo de canal está representado en los tres puntos de estudio (Fig. 2), cartografiados a partir de la fotografía aérea de 1978 (escala 1:18.000). Como en la mayoría de este tipo de ríos, se distingue un canal principal dentro de todo el modelo trenzado (RUST, 1972) y varios secundarios. 
GOMEZ Y MARTINEZ

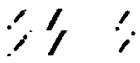

Fig. 1. Cuenca del río Oja.
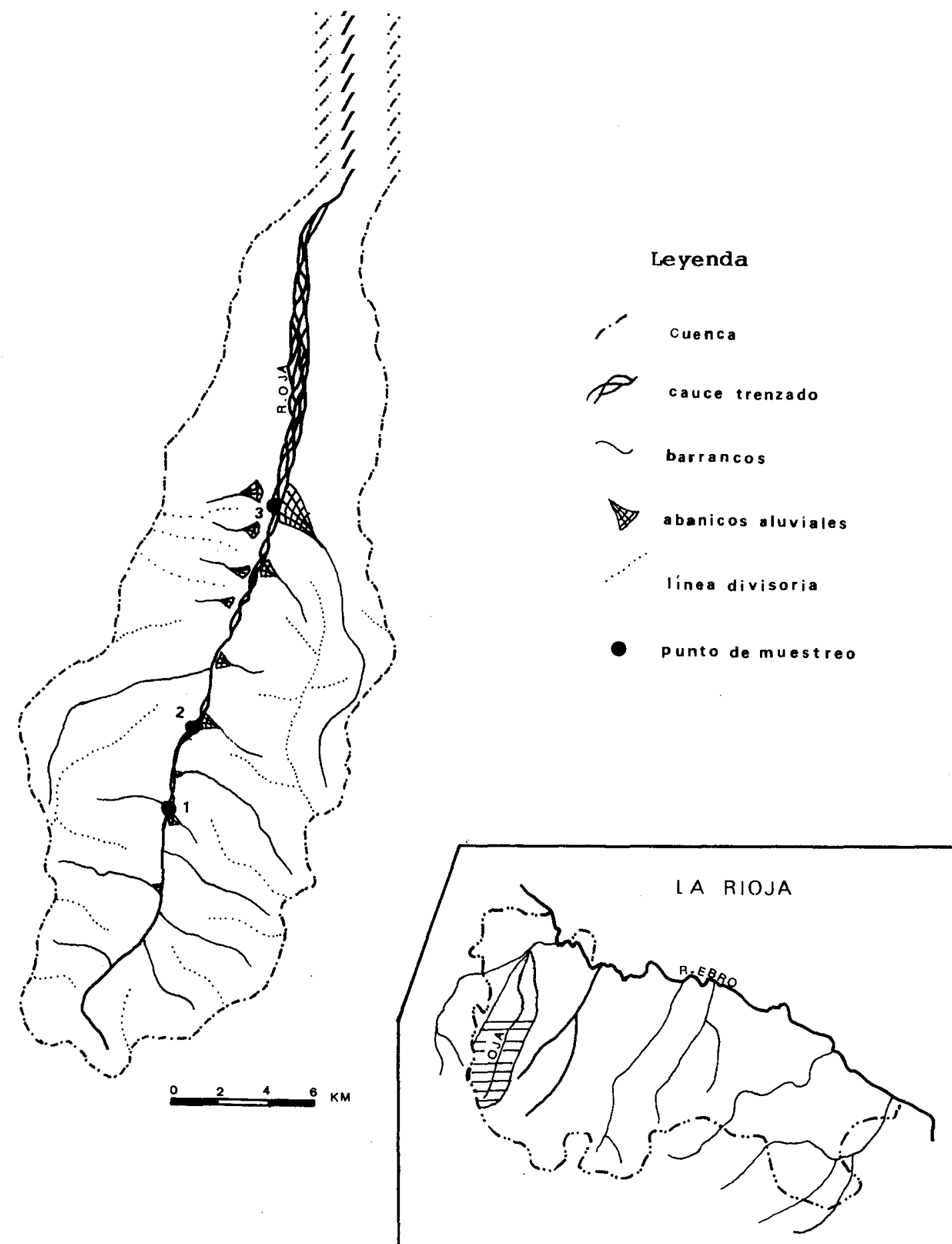

$0 \quad 15 \quad 30 \mathrm{kM}$ 


\section{ORGANIZACION DE SEDIMENTOS EN BARRAS}

Tabla 1. Algunas características morfométricas de los puntos de estudio en el cauce del río Oja.

\begin{tabular}{|c|c|c|c|}
\hline & Km. 12 & Km. 15 & Km. 25 \\
\hline Pendiente cauce $\left({ }^{\circ}\right)$ & $2 .^{\circ}$ & 1. $30^{\prime}$ & $1 .^{\circ}$ \\
\hline I. sinuosidad & 1,26 & 2,02 & 2,9 \\
\hline Anchura cauce (m) & 35 & 76 & 94,5 \\
\hline Tamaño Barras (m) & 41,2 & $\begin{array}{l}62 \\
21\end{array}$ & 96,5 \\
\hline Morfología barras & longitudinal & $\begin{array}{l}\text { longitudinal } \\
\text { puntual }\end{array}$ & $\begin{array}{l}\text { longitudinal } \\
\text { flujo trans. }\end{array}$ \\
\hline
\end{tabular}

El sector 1 (Km. 12) representa un modelo con un canal principal ligeramente ameandrado y con un índice de sinuosidad moderado $(1,26)$. La pendiente media del cauce en este punto es de casi $2 .^{\circ}$ (Tabla 1) y la anchura de $35 \mathrm{~m}$. En los sectores 2 y 3 el cauce muestra un trazado más rectilíneo aunque aumenta progresivamente el número de canales secundarios - tal como revelan los índices de sinuosidad (2,02 y 2,9 respectivamente) - a la vez que la anchura del cauce ( $76 \mathrm{~m}$ en el Km. 15 y 94,5 en el Km. 25), hecho constatado por CHORLEY, SCHUMM y SUDGEN (1984) para otros ríos trenzados, y disminuye la pendiente longitudinal hasta $10^{\circ} 30^{\prime}$ en la zona de Ezcaray y $10^{\circ}$ en Santurde.

En los tres puntos llegan al cauce conos de deyección, algunos de ellos semifuncionales y en el caso del cono de Santurdejo (sector 3) de grandes dimensiones. El río Oja, muy cargado ya de sedimentos y con escasa pendiente, tal como hemos señalado, es incapaz de desmontarlos, favoreciéndose aguas abajo un mayor desarrollo del entrecruzamiento de canales.

\section{RESULTADOS}

Los resultados obtenidos contribuyen a comprender algunos aspectos de las condiciones hidrodinámicas y características sedimentológicas del cauce del río Oja:

a) En el sector de Zaldierna (Km. 12) se han muestreado 8 puntos correspondientes a la barra longitudinal $-41,2 \mathrm{~m}$. de largo- y a los canales laterales. La barra se ha formado en el centro del cauce y ofrece en superficie una forma característica romboidal (Fig. 3a y Foto 1), limitada por canales activos.

Los histogramas de frecuencias reflejan el elevado ritmo de diseminación que presenta el tamaño medio de sedimentos (Fig. 4). El mayor número de intervalos de frecuencias se da en la punta de la barra (punto 1) y en los canales laterales (puntos 6 y 7), encontrándose bloques de más de $500 \mathrm{~mm}$. de eje mayor en los puntos 1 y 6 -más del $70 \%$ tiene tamaños entre 50 y $350 \mathrm{~mm}$.- junto a gravilla fina -materiales inferiores a $50 \mathrm{~mm}$. Pero mientras en el canal de la margen izquierda los tamaños predominantes son de 50 a $200 \mathrm{~mm}$., en el canal de la margen derecha, se concentran en las frecuencias de 100 a $300 \mathrm{~mm}$. Esto queda reflejado en el tamaño medio de los cantos: $217,06 \mathrm{~mm}$. en este punto frente a $130,65 \mathrm{~mm}$. en el canal secundario. La desviación estándar del calibre de los cantos en estos puntos tiene los valores más elevados de todo el sector. 
Fig. 2. Mapas de los tramos del cauce del río Oja donde se ha realizado el muestreo de cantos.

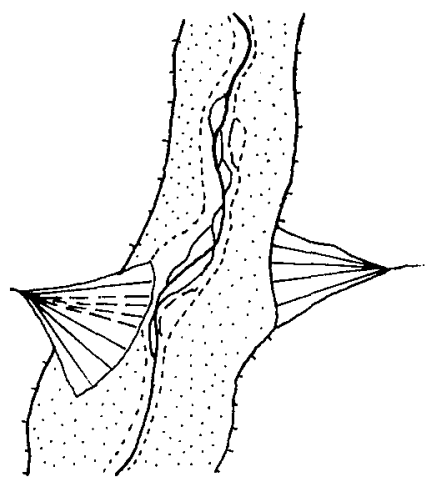

km 12
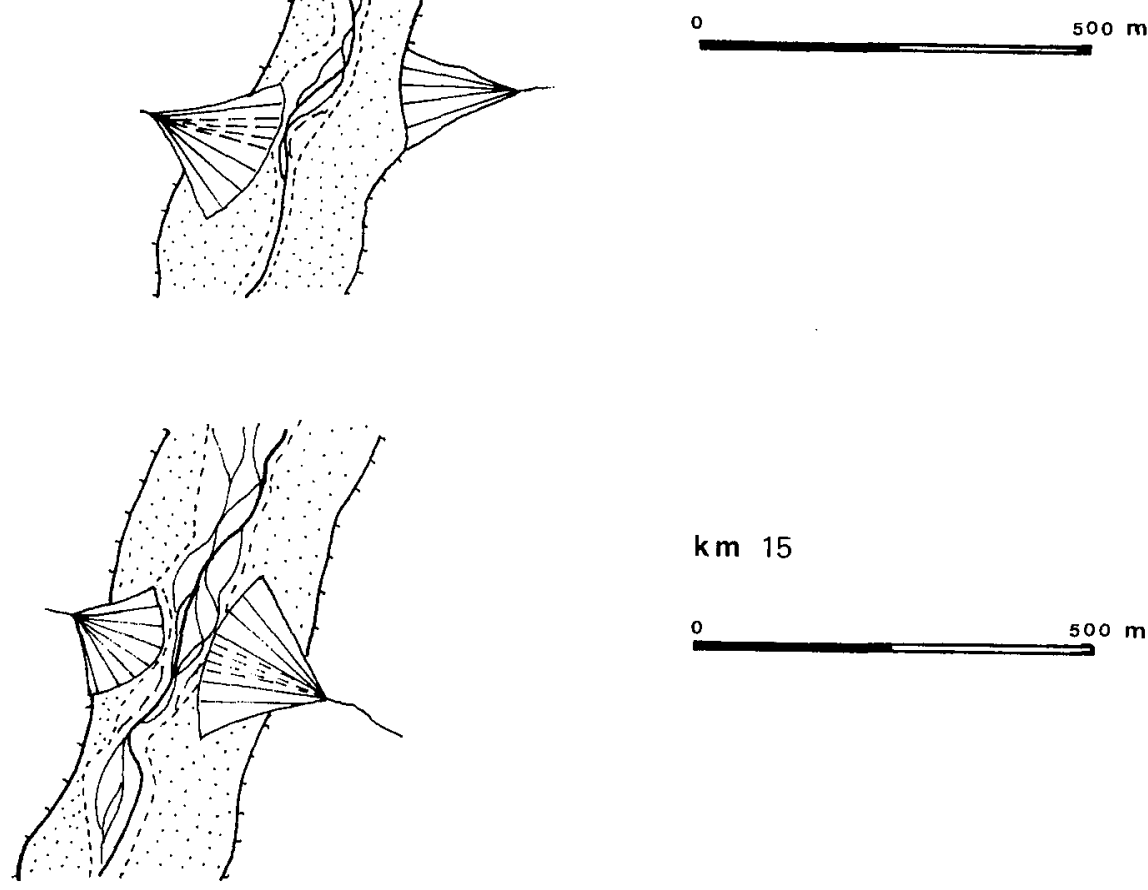

k m 15
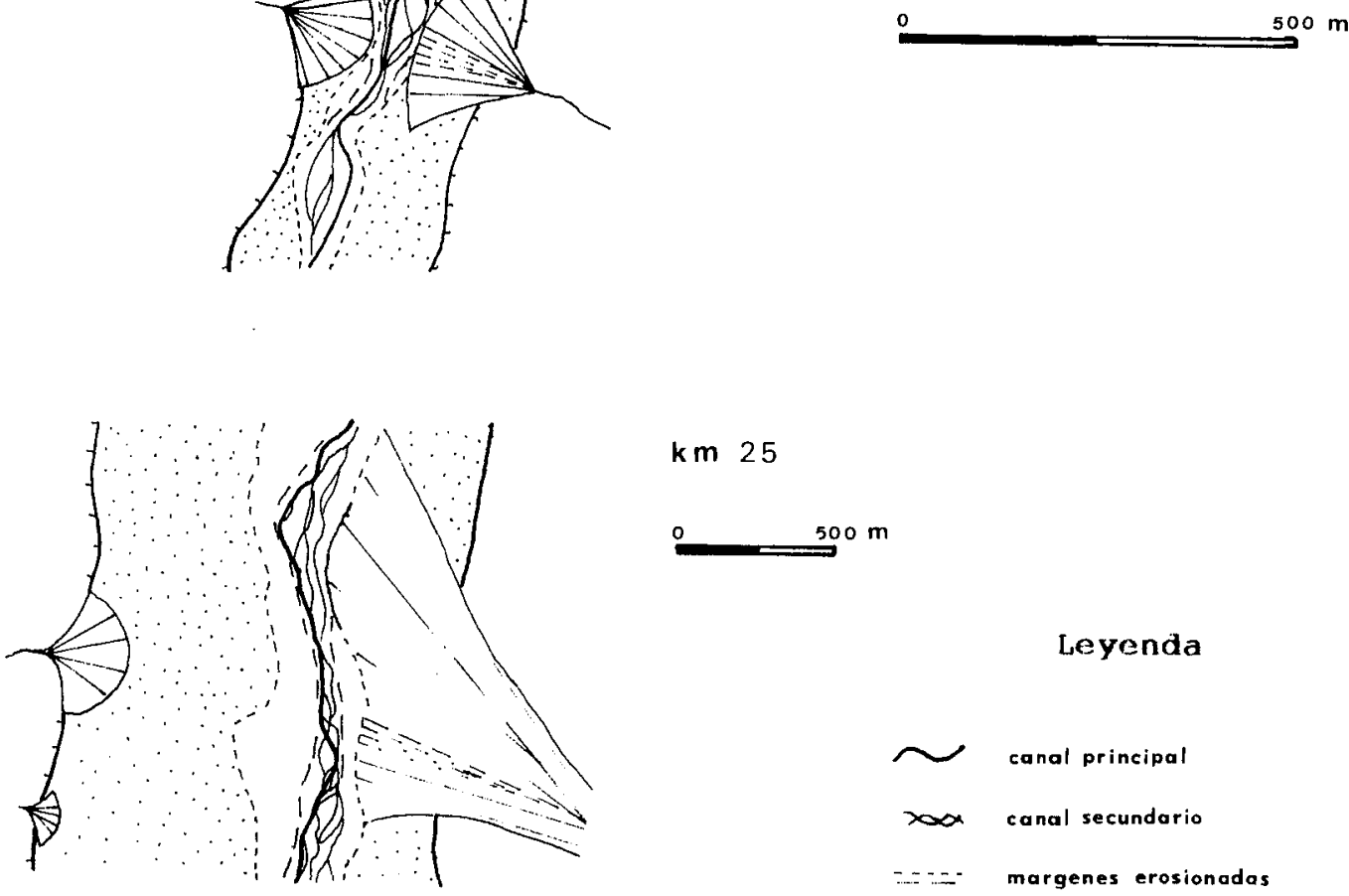

k m 25
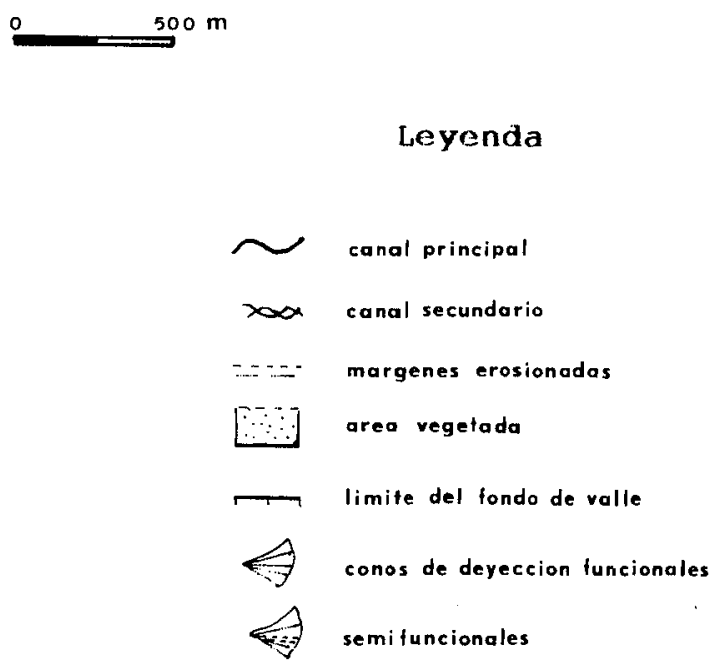
ORGANIZACION DE SEDIMENTOS EN BARRAS

Fig. 3. Morfología y secciones transversales del cauce en los tres sectores estudiados.
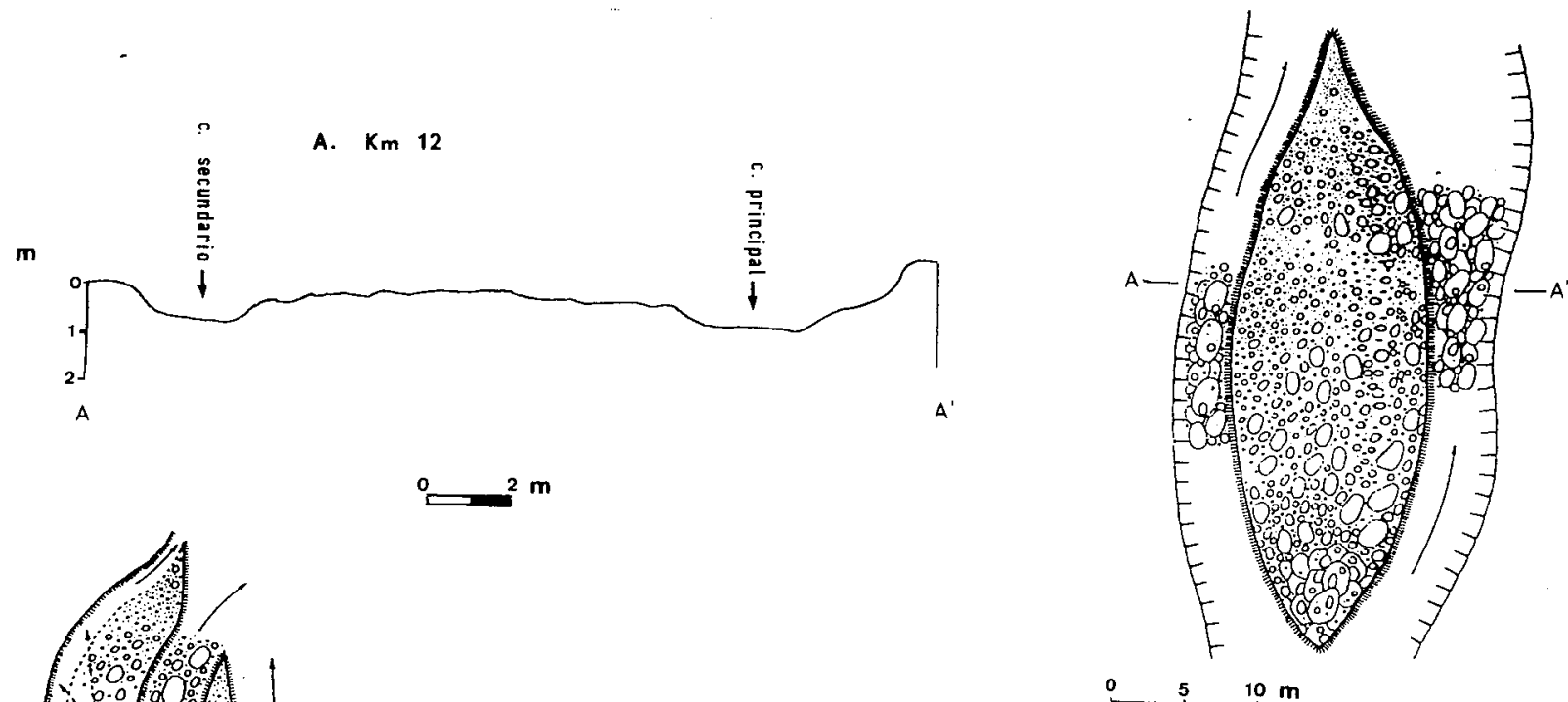

B. $\mathrm{Km} 15$
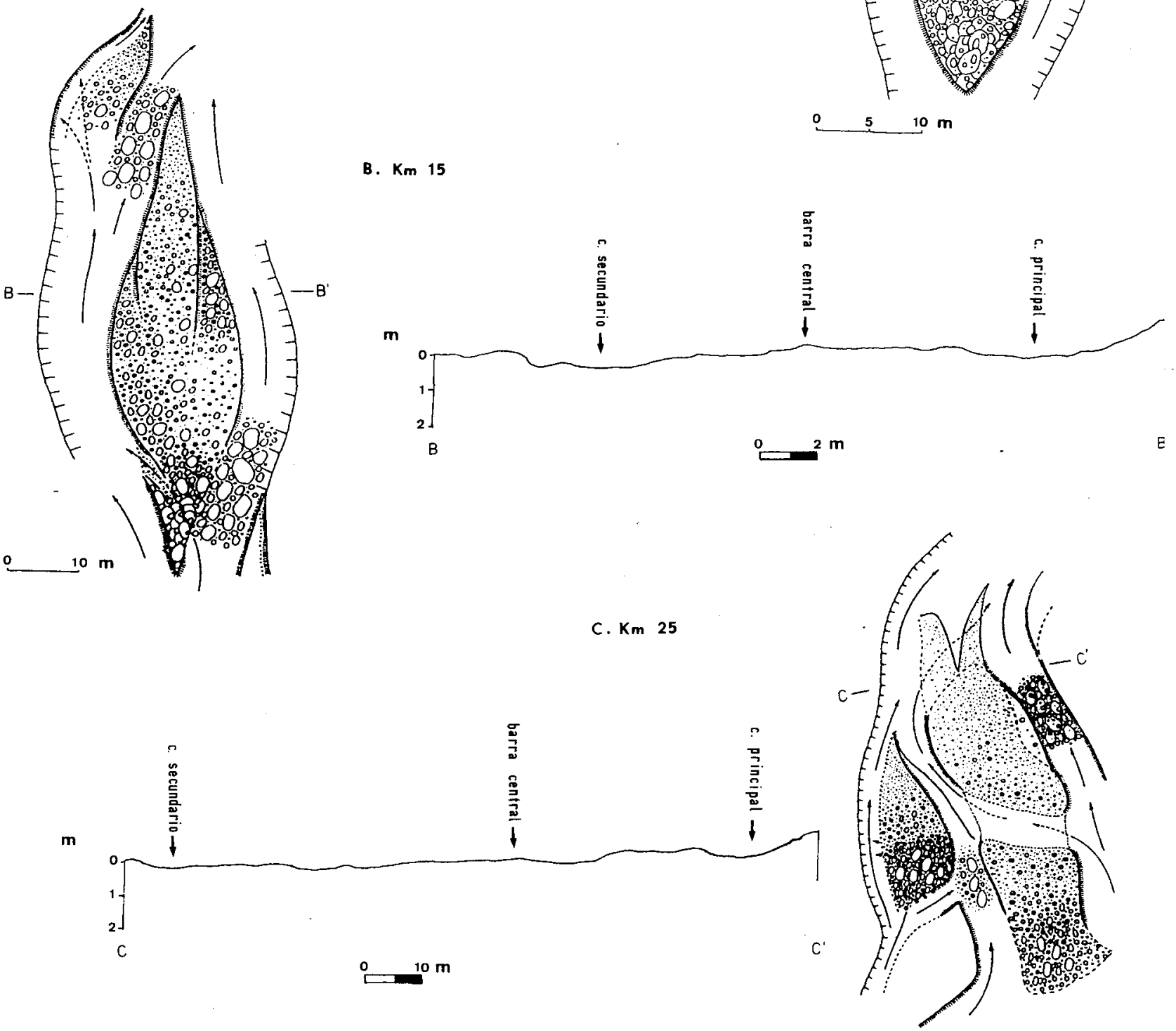

0 
GOMEZ Y MARTINEZ

Fig. 4. Histogramas de frecuencias del eje mayor de los cantos en el Km. 12 del cauce del río Oja.
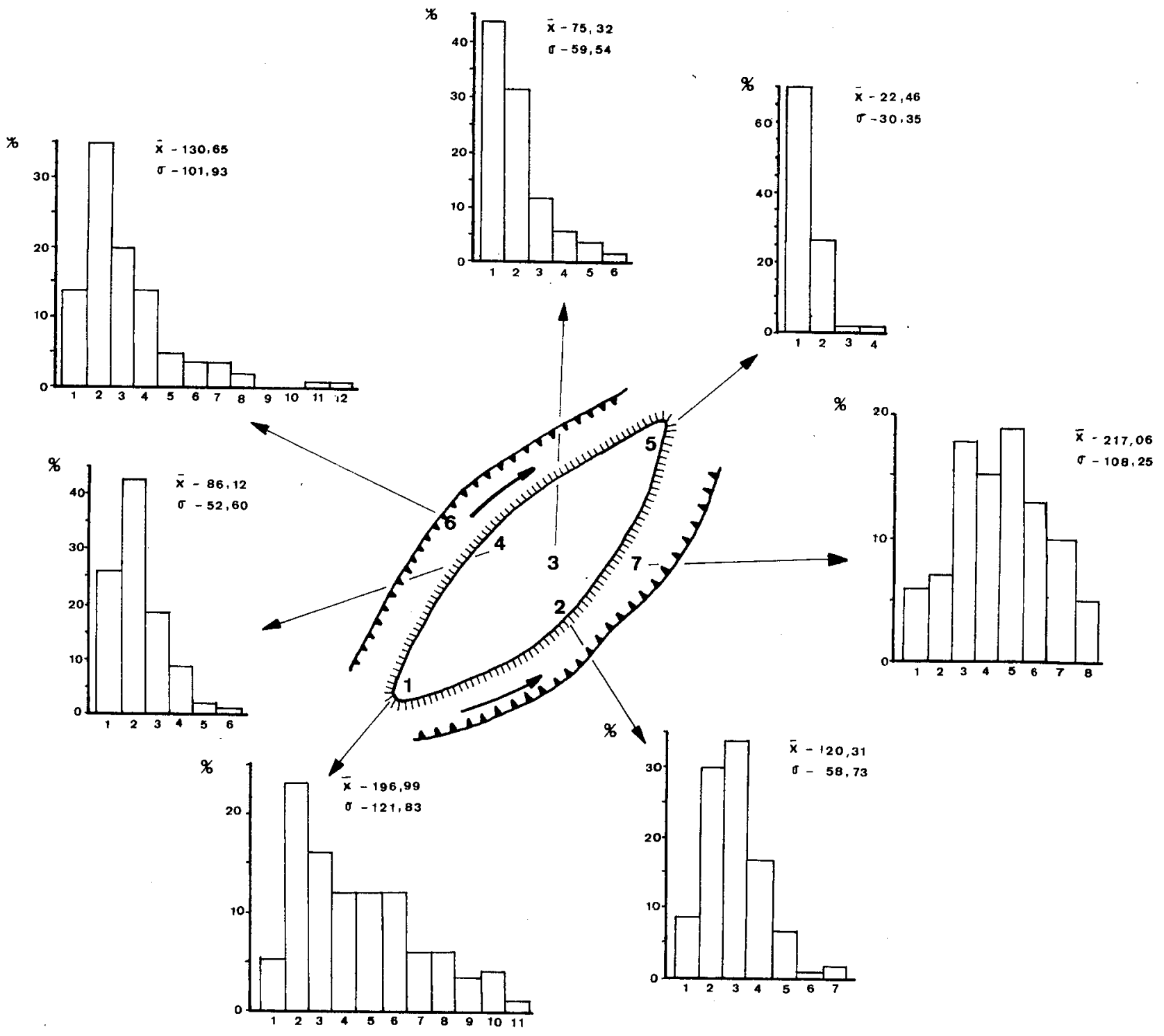
1. $0-50 \mathrm{~mm}$.
5. 200-250 mm.
9. $400-450 \mathrm{~mm}$.
2. $50-100 \mathrm{~mm}$.
6. $250-300 \mathrm{~mm}$.
10. $450-500 \mathrm{~mm}$.
3. $100-150 \mathrm{~mm}$.
7. $300-350 \mathrm{~mm}$.
11. $500-550 \mathrm{~mm}$.
4. $150-200 \mathrm{~mm}$.
8. $350-400 \mathrm{~mm}$.
12. $550-600 \mathrm{~mm}$. 


\section{ORGANIZACION DE SEDIMENTOS EN BARRAS}

Fig. 5. Histogramas de frecuencias del eje mayor de los cantos en el km. 15 del cauce del río Oja.

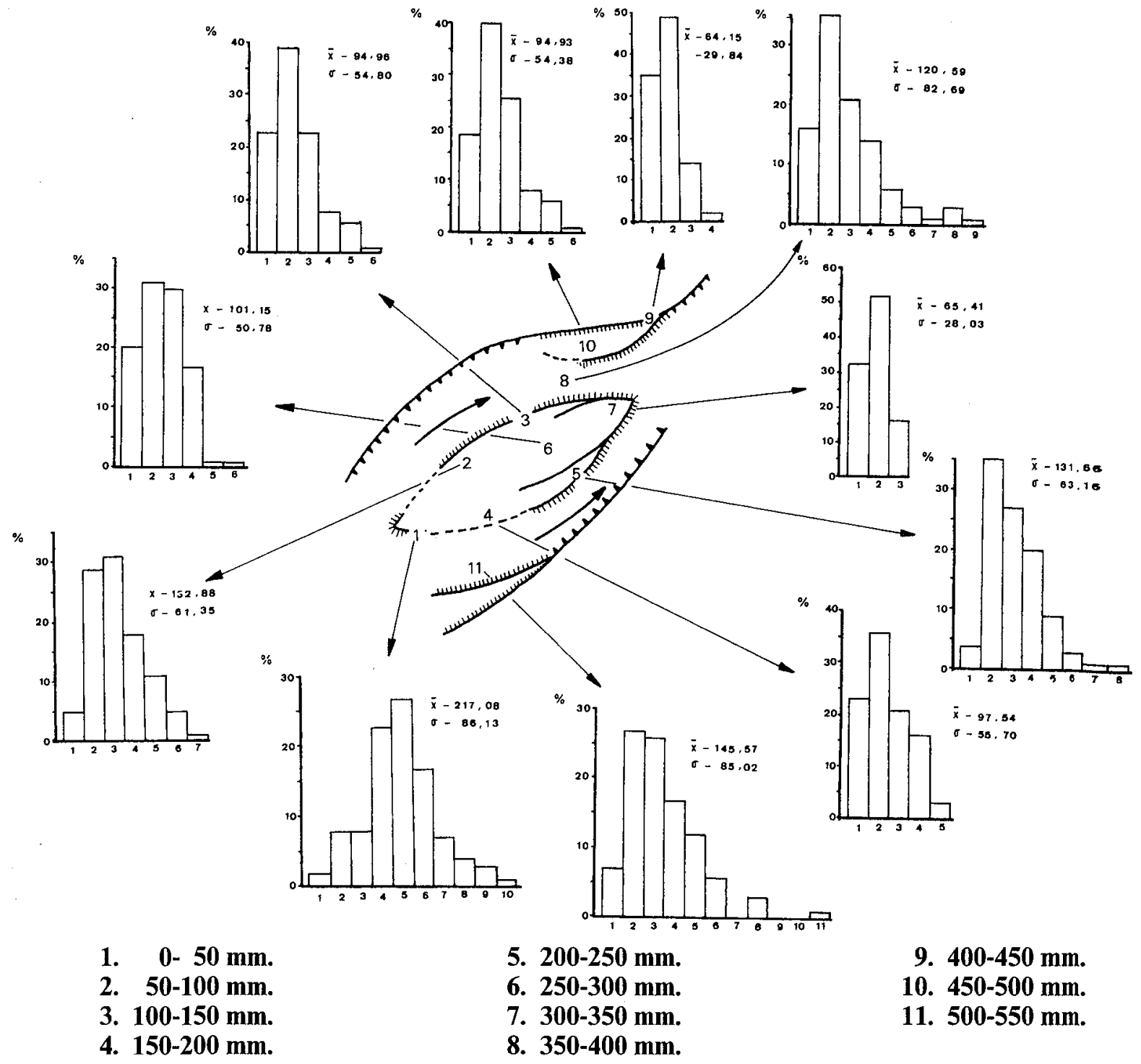

Una distribución más homogénea, con una desviación estándar similar, presentan el centro y márgenes laterales de la barra (puntos 2, 3 y 4). En los puntos 3 y 4 casi el $75 \%$ de los materiales tiene un tamaño comprendido entre 0 y $100 \mathrm{~mm}$. y ligeramente superior -entre 50 y $150 \mathrm{~mm}$.- la margen lateral derecha. El centro de la barra presenta un tamaño medio inferior al de las muestras adyacentes; se trata de una media muy matizada por la presencia de cantos de eje mayor inferior a $50 \mathrm{~mm}$. - un $45 \%$ del total muestreado.

El material de la cola de la barra es el de menor tamaño medio de las 26 localizaciones repartidas por las tres zonas de estudio -el $70 \%$ de los sedimentos tiene un tamaño inferior a $50 \mathrm{~mm}$.

Estas condiciones quedan reflejadas en la Fig. 3a. En el perfil transversal se distingue claramente los límites de las márgenes laterales de la barra, el canal principal, más ancho y profundo, con una pendiente local de $2 .^{\circ} 30^{\prime}$ y por lo tanto con mayor capacidad de transporte y el canal secundario, -que ha incidido menos en el lecho-, más estrecho y con una pendiente de 1..$^{\circ}$ 30. Es la barra más significativa topográficamente. Sus límites y morfología son precisos y su superficie no está recorrida por ningún flujo transversal o longitudinal.

b) En el Km. 15 -Fig. 3b y 5- se han muestreado 11 puntos pertenecientes a los canales, una barra central $-62 \mathrm{~m}$. de longitud- y a una barra puntual $-21 \mathrm{~m}$.- (SMITH, 


\section{GOMEZ Y MARTINEZ}

1978) localizada en el sector más convexo del cauce, siguiendo la curva del canal (GUPTA y DUTT, 1989); ofrece una superficie empinada -la pendiente en esta punta es casi de $2 .{ }^{\circ}$ frente a menos de $1 .^{\circ}$ aguas arriba- y está separada de la orilla por un canalillo (Foto 2). Ha sido generada durante un régimen de flujo bajo (SENGUPTA, 1974).

Destacan dos hechos en este sector:

Fig. 6. Histogramas de frecuencias del eje mayor de los cantos en el $\mathrm{Km} .25$ del cauce del río Oja

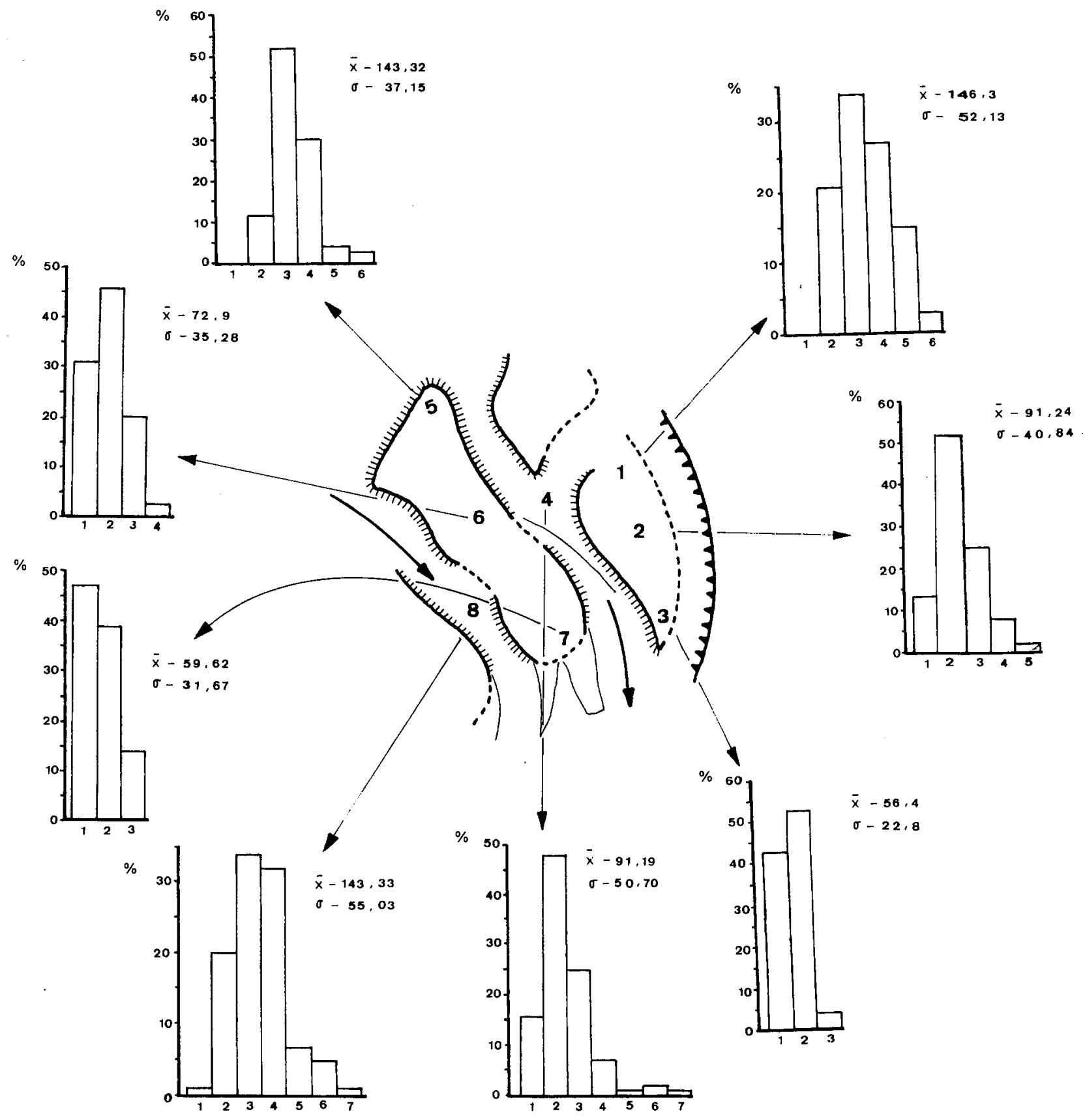

1. $\quad 0-50 \mathrm{~mm}$.

5. $200-250 \mathrm{~mm}$.

2. $50-100 \mathrm{~mm}$.

6. $250-300 \mathrm{~mm}$.

3. $100-150 \mathrm{~mm}$.

7. $300-350 \mathrm{~mm}$.

4. $150-200 \mathrm{~mm}$. 


\section{ORGANIZACION DE SEDIMENTOS EN BARRAS}

- La sensible reducción en el rango de los materiales muestreados con respecto al anterior sector. La presencia de cantos superiores a $450 \mathrm{~mm}$. sólo se da en el cauce principal (punto 11) que tiene una pendiente de $1 .^{\circ} 30^{\prime}$-superior a la del canal de la margen izquierda de la barra longitudinal- y supone tan sólo un $2 \%$. Esta tendencia se confirma con los valores más moderados que presentan las desviaciones estándar excepto para la punta de la barra y los canales laterales lo que nos informa de un mecanismo de clasificación del río aún incipiente; por otra parte son los puntos que mayor número de intervalos de frecuencias abarcan, con un predominio de cantos con tamaños entre 50 y $300 \mathrm{~mm}$.

- La gran similitud que presentan entre sí, tanto en la distribución por intervalos como en el tamaño medio, los cantos en la margen lateral izquierda de la barra central (punto 3) y los del sector central de la barra puntual (punto 10), así como las colas de las barras (puntos 7 y 9), donde el tamaño medio -en torno a $65 \mathrm{~mm}$. - y la desviación estándar se ha reducido notablemente -casi el $100 \%$ de los cantos tiene un tamaño inferior a $150 \mathrm{~mm}$. Este comportamiento se puede considerar lógico si se tiene en cuenta que todos ellos son ambientes deposicionales pertenecientes a un mismo canal y caracterizados por un cierto paralelismo. Los muestreos realizados en las márgenes laterales de las barras confirman que no existe una tendencia clara; sin duda, los límites poco definidos que la barra central presenta influye en sus características sedimentológicas (véase el perfil transversal en la figura $3 \mathrm{~b}$ ); asimismo, hacia la cola presenta dos niveles correspondientes a dos fases distintas: el superior, más antiguo, tenđría un origen deposicional y el más moderno podría deberse a una incisión de los canales.

Por otro lado, hay evidencias de que el sector más meridional de la barra central es invadido frecuentemente en periodos de aguas altas llegando a canalizarse y diseccionar la punta en la margen izquierda (Fig. 3b). Una situación similar tiene lugar en la barra puntual siendo muy difícil distinguir el límite entre ésta y el canal aguas arriba.

c) Mayor complejidad presenta aún el sector estudiado en el $\mathrm{Km}$. 25. Aquí se muestrearon dos barras ( 8 puntos en total) pertenecientes junto con otras colaterales a un sistema deposicional mayor que ha sido diseccionado y desmantelado parcialmente por flujos transversales (Foto 3) dando lugar a un perfil del cauce muy homogéneo (Fig. 3c) sin contrastes bruscos entre los límites de las barras y los canales, menos profundos y pendientes - menos de $1 .^{\circ}-$ que en los sectores anteriores. La gran anchura que en este tramo presenta el cauce y, en consecuencia, el mayor desarrollo en superficie de los depósitos, la imposibilidad del canal para incidir y el hecho de que deriven de una estructura anterior, parecen ser las causas de esta mínima relevancia. La gradación entre barras y canales sólo se ve interrumpida por el desarrollo de márgenes erosionadas en el sector más antiguo de la barra.

En ambas barras muestreadas se constata el decrecimiento del tamaño de los materiales desde la punta a la cola $-80 \mathrm{~mm}$. de diferencia-, tal como nos revela el tamaño medio, aunque considerando que son barras de gran desarrollo longitudinal -casi $100 \mathrm{~m}$.- el ritmo de variación es muy lento y poco significativo. Se trata de un material muy seleccionado y los tamaños se concentran en muy pocos intervalos de frecuencias $\mathrm{y}$, de hecho, no hay ningún canto en los 8 puntos muestreados que supere $105350 \mathrm{~mm}$. Es significativo que en las puntas de las barras no hay materiales inferiores a $50 \mathrm{~mm}$. y más del $75 \%$ de los cantos está incluido en los intervalos de 100-200 mm. En las colas de las barras hay predominio absoluto de cantos inferiores a $100 \mathrm{~mm}$. -el $90 \%$ - El comportamiento de la desviación estándar mantiene la tendencia apuntada más arriba, presentando los valores más bajos de los 3 sectores estudiados.

Se observan dos hechos significativos:

- La afinidad granulométrica de la punta de la barra más pequeña (punto 1) con la punta de la barra más grande (punto 5) en lugar de con la zona media de la misma, con la 


\section{GOMEZ Y MARTINEZ}

cual debería corresponderse por su situación dentro del cauce teniendo en cuenta que ambas pertenecen a una macroestructura.

- La barra central presenta asociadas, sin solución de continuidad con el material que la forma, acumulaciones de sedimentos, en forma de espigones, mucho más finos (Foto 4).

\section{DISCUSION DE RESULTADOS}

Con frecuencia se ha definido a las barras como "almacenes» de sedimentos (CHURCH y JONES, 1982; JAEGGI, 1987). Según esta interpretación, su formación es consecuencia de las condiciones locales de flujo (LEOPOLD et al, 1964) relacionadas a su vez con el tamaño de los sedimentos aportados en ese punto. Atendiendo a esta premisa, es de esperar que la barra, entendida como un ambiente donde predomina la deposición, refleje fielmente las condiciones de flujo que dieron lugar a su formación. No obstante, la misma barra introduce nuevos elementos en el régimen hidráulico de la zona donde se instala -bifurcación del caudal, aumento de la resistencia al flujo, ensanchamiento del canal, etc.cuya consecuencia inmediata es la generación de alteraciones aún más localizadas que pueden llegar a enmascarar la situación que constituyó su origen. Estas alteraciones en el régimen hidráulico son las responsables de la creación de microambientes deposicionales.

El presente estudio se basa en el hecho constatable de que cada medio sedimentario puede caracterizarse de acuerdo con la textura de sus sedimentos (SANJAUME et al, 1985). A pesar de la gran estabilidad que estas estructuras presentan bajo condiciones normales de flujo, los materiales que las componen no permanecen inalterables, sino que evolucionan in situ mediante mecanismos de selección y expulsión (BLUCK, 1982) que reorganizan superficialmente los sedimentos eliminando diferencias bruscas de granulometría. En otras ocasiones, acumulaciones de sedimentos sensiblemente distintos, más pequeños, y pertenecientes a una fase deposicional diferente, permanecen adosados a la barra hasta su eliminación o reorganización. Estas situaciones no suponen necesariamente un inconveniente en el análisis sedimentológico, sino que son consecuencia de la propia dinámica fluvial $\mathrm{y}$, por lo tanto, constituyen elementos de la misma que deben ser tenidos en cuenta.

La evolución de los rasgos sedimentológicos de los tres sectores estudiados responde a diversos ajustes propiciados por la dinámica fluvial de manera que podemos referirnos a una tendencia general que siguen tanto el tamaño de los cantos como la morfología de las barras, con una serie de irregularidades favorecidas por la propia dinámica interna del río Oja, como ya hemos indicado:

- El material más grosero y el mayor número de intervalos de frecuencias de tamaños se localiza en las puntas de las barras: el simple choque de los cantos arrastrados contra el depósito significa su inmediata deposición a la vez que constituyen un elemento de dispersión (LEOPOLD et al, 1964; 1966); sin embargo, la existencia de elementos mucho más pequeños exige una explicación basada en el comportamiento hidráulico del flujo. En efecto, la punta de la barra es invadida por el agua en momentos de crecida -indicando por otra parte la deposición de un flujo de elevada energía (GUPTA y DUTT, 1987)-: los sedimentos transportados en suspensión, ante el cese de la fuerza tractora, se depositan, introduciéndose entre los intersticios dejados por los cantos más gruesos llegando incluso a su colmatación. Esta misma circunstancia impide la posterior remoción de las partículas al quedar protegidas por los materiales de mayor tamaño. Es el efecto de «hiding» descrito por ANDREWS y PARKER (1987).

- El hecho de que los sedimentos del canal muestren un calibre superior al resto de los puntos muestreados en la barra concuerda con los resultados obtenidos por SANJAUME et al (1985) para cursos de régimen hidrológico distinto. Por otra parte, se ha establecido una 


\section{ORGANIZACION DE SEDIMENTOS EN BARRAS}

jerarquía de canales atendiendo al tamaño de los materiales: el canal principal tiene cantos de calibre superior a los secundarios y es, por otra parte el más ancho, profundo y pendiente y por lo tanto el que presenta una mayor competencia de transporte y un mecanismo de clasificación incipiente.

- Prácticamente en todos los casos el tamaño medio de materiales en el centro de las barras es sensiblemente inferior al de los muestreados en las márgenes laterales de las mismas. El centro de la barra resulta más inaccesible a las aguas de avenida -es la zona topográficamente más elevada- por lo que no actúan aquí los mecanismos de selección y expulsión. El lavado del material más fino sí ocurre en los laterales, más expuestos a los caudales altos. Los límites mal definidos que la barra presenta y que ya hemos señalado anteriormente - casos de Ezcaray y Santurde - también influyen en la organización superficial de los sedimentos.

- Los sedimentos más finos se depositan en la cola de la barra, de acuerdo con la gradación del tamaño de materiales desde la punta a la cola; es evidente que aguas abajo se tiende a una mayor homometría de los cantos, producto de las condiciones de transporte - muy diezmadas por la bifurcación del caudal y el aumento de la resistencia- que da lugar a la deposición de materiales cada vez más finos provocada por el «remanso» ocasionado en la confluencia de los flujos; esta tendencia está muy bien definida en el Km. 11 (Zaldierna) que presenta el tamaño medio más bajo de cantos para todos los puntos muestreados en los tres sectores. Si atendemos a los modelos de crecimiento establecidos, la existencia de un material tan pequeño $(22,5 \mathrm{~mm}$.) en la cola de la barra respondería a una fase de evolución más avanzada que el resto de las barras estudiadas.

- Las irregularidades observadas en el Km. 25 tienen una doble explicación: la no afinidad granulométrica entre los distintos puntos muestreados dentro de las barras que pertenecen a otra forma deposicional mayor está relacionada con los mecanismos de selección y expulsión que, como describe BLUCK (1982), dan lugar a un aumento local del tamaño medio, acentuando el salto granulométrico que caracteriza a las barras mejor definidas. La existencia de flujos superficiales responsables de este lavado ha sido comprobada (Foto 3). Por otra parte, las acumulaciones de material más fino adosadas a la cola en forma de espigones corresponden a condiciones sedimentarias muy particulares, relacionadas con un flujo de baja energía con unas condiciones de transporte muy mermadas y sin duda, diferentes a las que dieron lugar a los depósitos mayores. No es probable que perduren en la próxima crecida.

Para concluir señalaremos que son varios los factores que intervienen en la evolución del tamaño de los cantos a lo largo del curso de un río. A gran escala, y como ya pusieron de manifiesto GARCIA-RUIZ et al, (1987) para el mismo río Oja, es evidente que el descenso de la pendiente y el alejamiento de las áreas productoras de sedimentos dan lugar a una progresiva disminución en el tamaño de los cantos. Pero esta reducción no se limita sólo al canal, sino que se transmite a los diferentes ambientes deposicionales, claro reflejo de las condiciones locales de transporte. En la misma dirección se encuentra la reducción de tamaño de los sedimentos dentro de un mismo ambiente deposicional. Todas las barras analizadas han mostrado esta reducción desde el extremo aguas arriba -punta- hasta el extremo aguas abajo -cola-, reducción impuesta por las alteraciones que en el régimen hidráulico introduce la propia existencia de la barra.

Asimismo, las características granulométricas y sedimentológicas de las barras están mejor definidas en el tramo alto del curso $-\mathrm{Km}$. 12-, donde los materiales presentan una clasificación muy pobre y donde la granulometría del canal y barra presenta diferencias acusadas. Aguas abajo aquellas características se atenúan progresivamente y tienen en el $\mathrm{Km}$. 25 (Ezcaray) un claro ejemplo: límites mal definidos, morfología confusa, topografía poco acusada, presencia de flujos superficiales, etc. Por otro lado, al mecanismo de selección que 


\section{GOMEZ Y MARTINEZ}

ha desarrollado el propio río y que le impide el acarreo de los cantos más gruesos se une el hecho de que en este sector el cauce recibe una carga muy selectiva procedente de los conglomerados de borde de cuenca, de origen fluvial, reflejado en el escaso número de intervalos de frecuencias y en los valores muy bajos de las desviaciones estándar.

\section{BIBLIOGRAFIA}

ALLEN, J.R.L., 1965. A review of the origin and characteristics of recent alluvial sediments. Sedimentology, 5: 89-191.

Andrews, E.D. and PARKer, G., 1987. The corase surface layer as a response to gravel mobility. In: Thornes, C.P., Bathrust, J.C., Hey, R.D. and Simons, D.B. (Eds), Problems of sediment transport in gravel bed rivers, Wiley Interscience.

BLUCK, B.J., 1974. Structure and directional properties of some valley sandur deposits in southern Iceland. Sedimentology, 21: 533-554.

BLuCK, B.J., 1982. Texture of gravel bars in braided rivers. In: Hey, R.D., Bathurst, J.C. and Thorne, C.R. (Eds). Gravel-bed Rivers. John Wiley y Sons Ltd. pp: 339-355.

Chorley, R., Schumm, S.A. and Sugden, D.E., 1984. Geomorphology. Methuen, 605 pp. London.

Church, M. and Jones, D., 1982. Channel bars in gravel bed rivers. In: Hey, R.D., Bathurst, J.C. and Thornes (Eds). Gravel-bed Rivers. John Wiley y Sons Ltd. pp: 291-338.

Dawson, M., 1988. Sediment size variation in a braided reach of the Sunwapta river, Alberta, Canadá. Earth Surface Processes and Landforms, 13: 599-618.

Doeglas, J., 1962. The structure of sedimentry deposits of braided streams. Sedimentology, 1: 167-193.

DunNe, T. and LeOPOLD, L.B., 1978. Water in environmental planning. Freeman and Co. 818 pp. San Francisco.

FAHNESTOCK, R.K., 1963. Morphology and hidrology of a glacial stream: White River, Mount Rainier, Washington. U.S. Geological Survey Professional Paper, 422-A: 1-67.

Garcia-Ruiz, J.M., Gomez-VILlar, A. y Ortigosa IzQuierdo, L.M., 1987. Aspectos dinámicos de un cauce fluvial en el contexto de su cuenca: El ejemplo del rio Oja. Monografías del Instituto Pirenaico de Ecología (Jaca) e Instituto de Estudios Riojanos (Logroño). 112 pp.

Gregory, K.J. and Walling, D.E., 1976. Drainage basin. Form and process. Ed. Arnold, 458 pp. London.

Gupta, A. and Dutr, A., 1989. The Auranga: description of a tropical monsoon river. Z. für Geomorphology, 33 (1): 73-92.

JAEGGI, M.N.R., 1987. Interaction of bed-load transport with bars. In: Thornes, C.P., Bathurst, R.D. and Hey, R.D. (Eds). Sediment transport in gravel bed rivers. John Wiley y Sons. Ltd. pp: 829-841.

Krigstrom, A., 1962. Geomorphological studies of sandur plains and their braided rivers in Iceland. Geografiska Annaler, 44: 328-346.

Leopold, L.B. and Wolman, M.G., 1957. River channel patterns: braided, meandering and straight. U.S. Geological Survey Professional Paper, 282 pp. 


\section{ORGANIZACION DE SEDIMENTOS EN BARRAS}

Leopold, L.B., Wolman, M.G. and Miller, J.P., 1964. Fluvial processes in Geomorphology, Freeman, 521 pp. San Francisco.

Miall, A., 1977. A review of braided river depositional environment. Earth Science Reviews, 13:1-62.

RiBA, O., 1955. Sobre la edad de los conglomerados terciarios del borde $\mathrm{N}$ de la Sierra de la Demanda y Cameros. Notas y Comunicaciones IGME, 39: 39-50. Madrid.

Rust, B.R., 1972. Structure and processes in a braided river. Sedimentology, 18: 221-245.

Sanjaume, E., Segura, F. y Meyer, M.J., 1985. Estudio sedimentológico de la Rambla de Chiva. Cuadernos de Investigación Geográfica 11: 125-136. Logroño.

SEgura, F., 1987. La influencia del armouring sobre las características de los sedimentos de algunas ramblas del País Valenciano. X Congreso Nacional de Geografía (A.G.E.). Volumen I: $81-91$.

Sengupta, S., 1974. Fluviatile cross-stratification -ancient, recent and experimental. Quart. J. Geol. Mining and Metal Soc. India, 46: 87-99.

ScHumm, S.A., 1968. Speculations concerning paleohydrologic controls of terrestial sedimentation. Geological Society of American Bulletin, 79: 1.573-1.588.

SMITH, N.D., 1978. Some comments on terminology for bars in shallow rivers. In: Fluvial Sedimentology. Miall, A.D. (Ed). Canadian Society of Petroleum Geologists Memoir, 5: 85-88.

Strahler, A., 1956. The nature of induced erosion and aggradation. In: W.L. Thomas Jr (Ed) Man's role in changing the face of the Earth. The University of Chicago Press, pp: 621-638. Chicago.

Wolman, M.G. 1954. A method of sampling coarse river-bed material. Trans. Amer. Geophis., Union, 35: 951-956. 


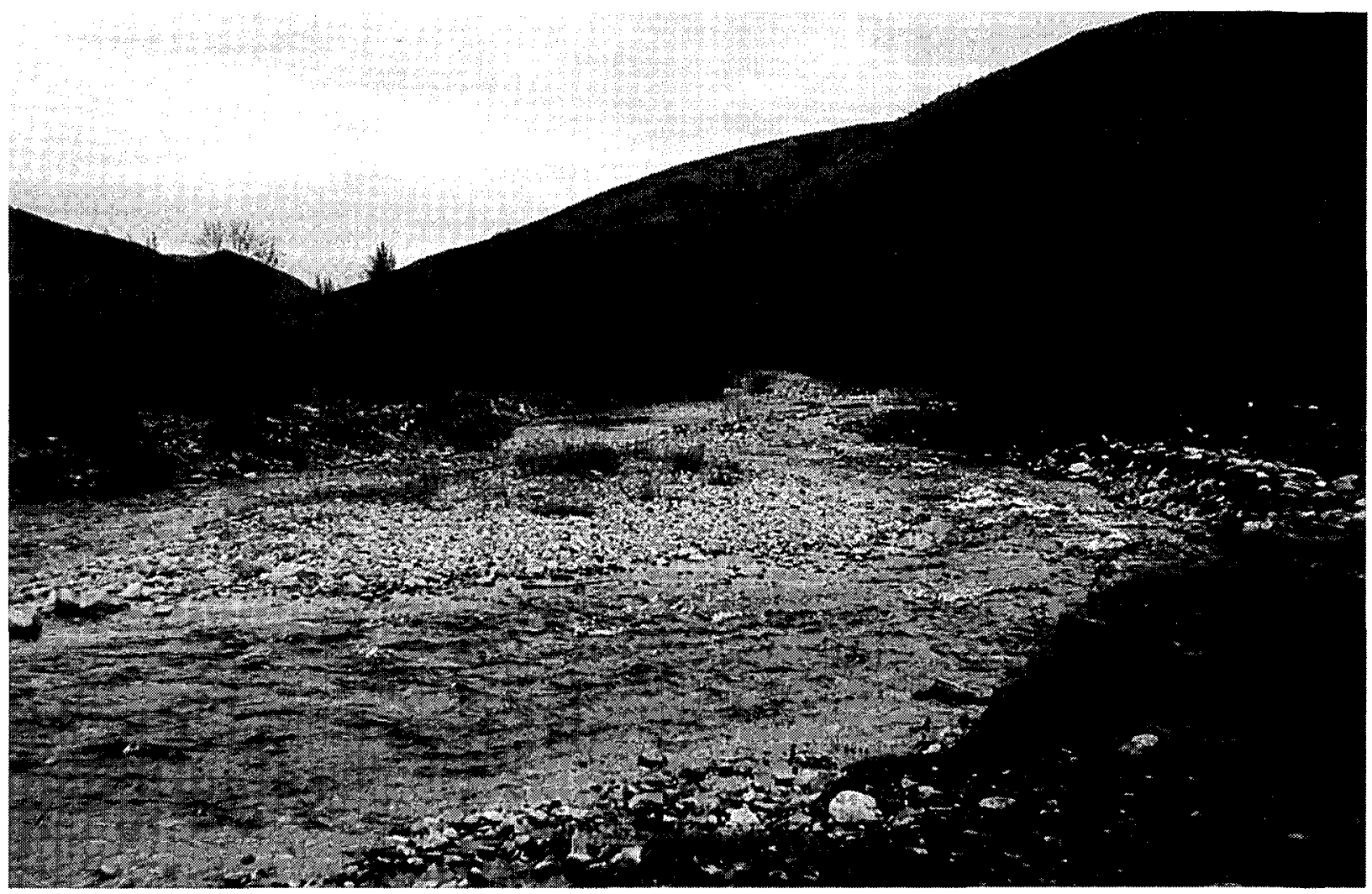

Foto 1. Km. 12 Sección del cauce. Destaca la forma romboidal de la barra central

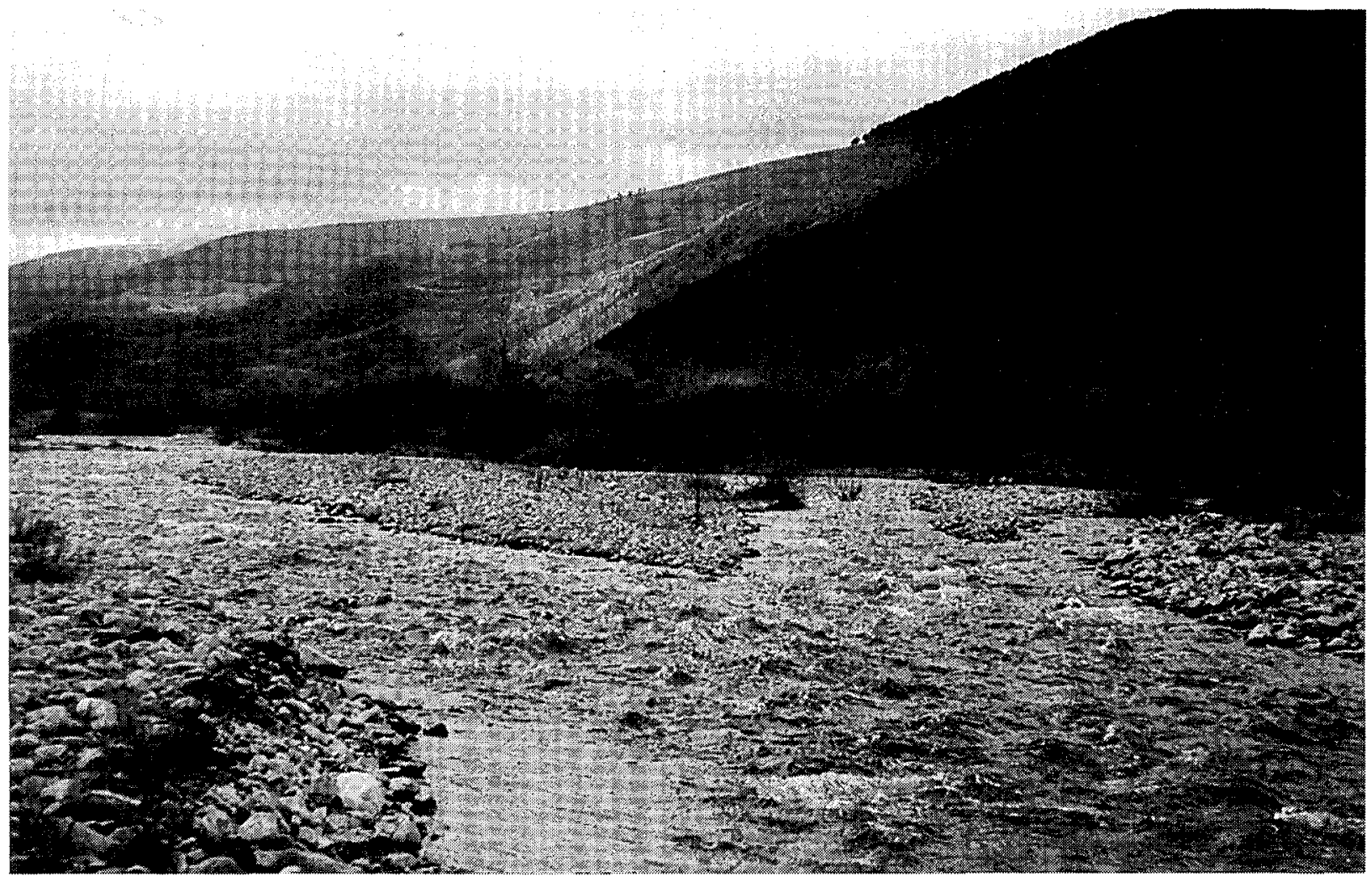

Foto 2. Vista parcial del cauce del río Oja en el $\mathrm{Km}$. 15. Se observa parte de la barra central y la barra puntual 


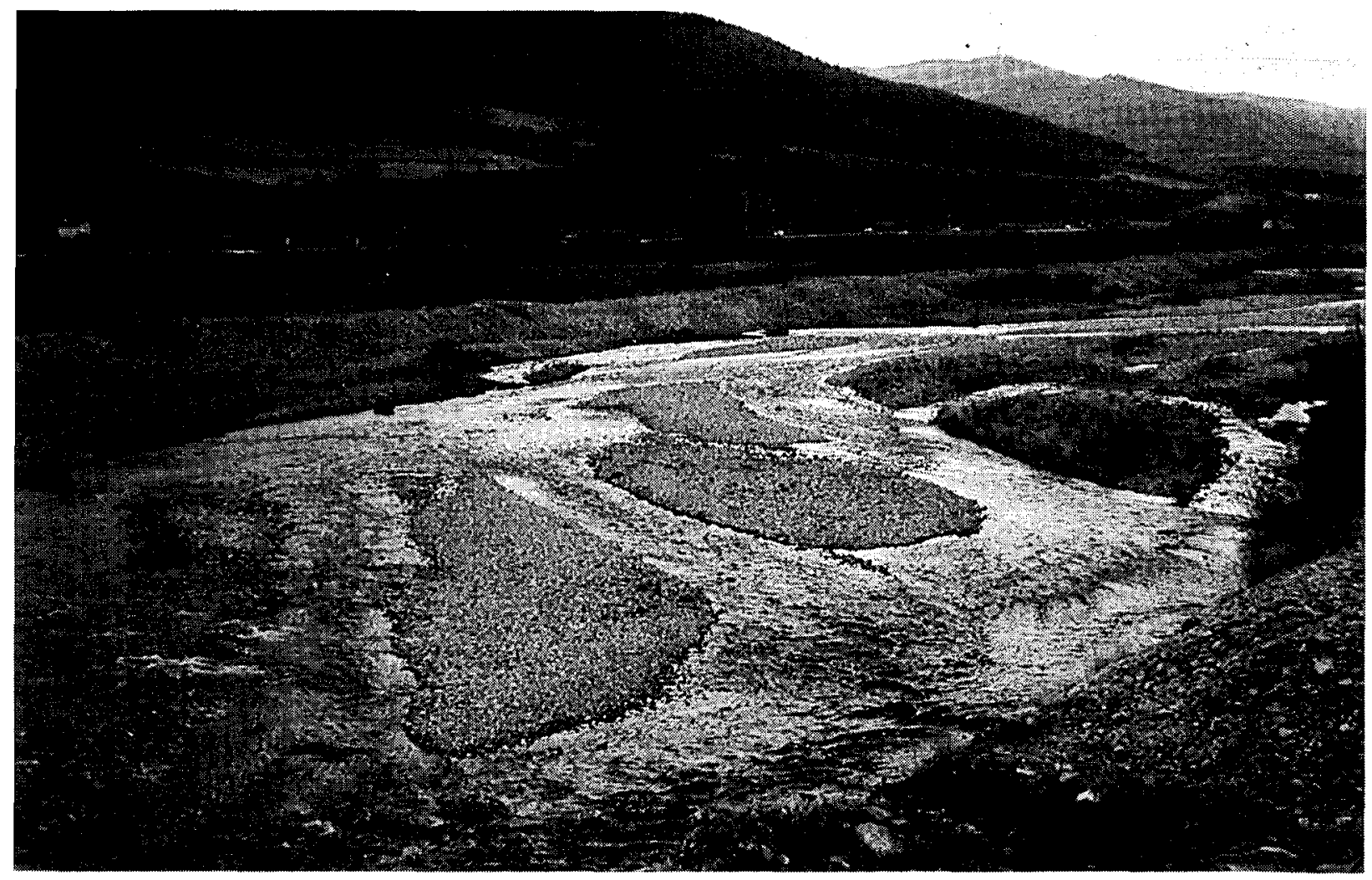

Foto 3. Cauce del río Oja en el Km. 25. Barra longitudinal diseccionada por flujos transversales

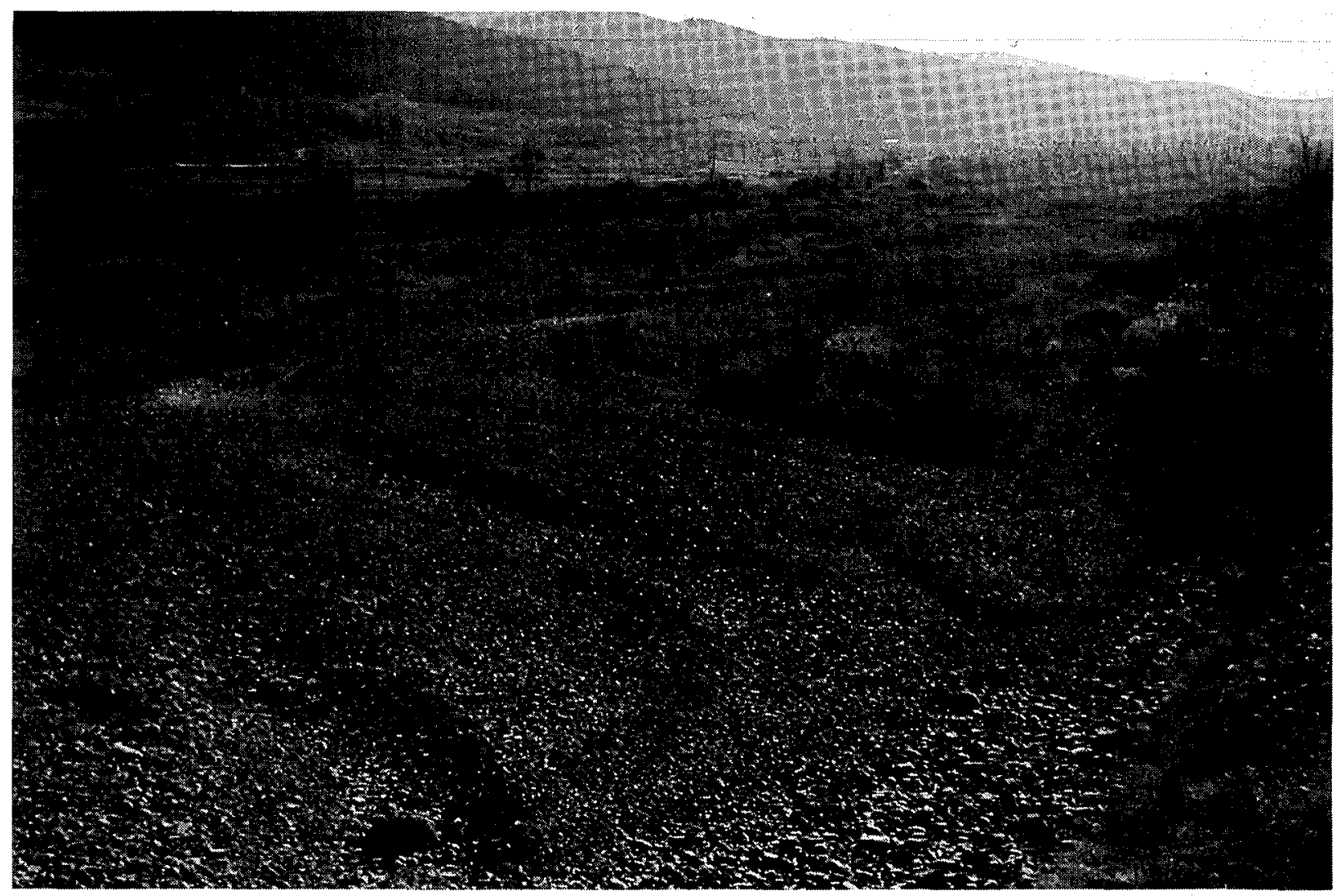

Foto 4. Acumulaciones de sedimentos más finos adosados a la cola de la barra en el Km. 25 
